\title{
2280. Optimization of the control scheme for human extremity exoskeleton
}

\author{
Yang $\mathrm{Li}^{1}$, Cheng $\mathrm{Xu}^{2}$, Xiaorong Guan ${ }^{3}$, Zhong $\mathrm{Li}^{4}$ \\ School of Mechanical Engineering 105, Nanjing University of Science and Technology, \\ Nanjing, China \\ ${ }^{3}$ Corresponding author \\ E-mail:1562339471@qq.com, ${ }^{2 x u c h e n g 62 @ m a i l . n j u s t . e d u . c n, ~}{ }^{3}$ guanxr@vip.163.com, \\ 4zhong0814@163.com
}

Received 12 July 2016; received in revised form 12 October 2016; accepted 25 November 2016

DOI https://doi.org/10.21595/jve.2016.17397

\begin{abstract}
One kind traditional PI (Proportion Integration) control scheme was proposed to be applied to human extremity exoskeleton (HEE, for short) in our previous works, and the simulation study and experimental study was also carried out. By analyzing the simulation calculation results, it was shown that the traditional PI controller is not very appropriate for human body in every movement speed condition. So, at last the fuzzy self-adaptive PI controller was proposed to solve this problem. Eventually, the superiority and feasibility of the fuzzy selfadaptive PI controller was proved by the simulation results and experimental results.
\end{abstract}

Keywords: human extremity exoskeleton, simulation study, experimental study, fuzzy self-adaptive PI controller.

\section{Introduction}

HEE is an electromechanical structure worn by operator and matching the shape and functions of human body [1]. It merges the machine power and the human intelligence in order to enhance the intelligence of the machine and to power the operator [2].

The "BLEEX" developed by University of Berkeley of the United States [3-5], the "HAL" developed by Tsukuba University of Japan [6], the "Boyets-21" developed by the third central institute of the Russian defense ministry [1], the "ReWalk" developed by Argo Medical Technologies company of Israel [7] and the "NTU" developed by Nanyang Technological University of Singapore [8] are the most famous HEE in the word. However, some of them are used for medical purposes, and the effects of the control schemes of those military exoskeletons are still not very good.

Besides, in 2015, there are more than 15 research institutions of China attended the HEE competition which was organized by the general armaments department of China. The competition lasted three days, and the champion was won by the prototype developed by 208 Research institute of China South Industries Group Corporation, Harbin institute of technology and Nanjing University of science and technology $[9,10]$. It can be observed according to the competition result that the military HEEs developed by Chinese institutions are still in the phase of development. And according to the feeling of the wearer, although most of those HEEs can barely achieve synchronous walking with human bodies, the posture of walking will become unnatural. Besides, only the knee joints of the prototypes of champion and runner-up [1] are controlled, this will make the difficulty of designing good man-machine coordination control strategy greatly reduced. However, it is conceivable that the power support effect of the champion and runner-up prototypes is low. So, in general, the control scheme is the most important factor that needs to be improved.

So, in this paper, the fuzzy self-adaptive controller was proposed to optimize the traditional PI controller which was proposed in our previous works.

\section{Previous work}

Force control method got more and more attention of humanoid robot researchers recently 
[11]. So, in our previous works, it was also adopted by us to design the control scheme of HEE. And the simulation study and experimental study were carried to verify the design of the whole electromechanical system of HEE [12].

\subsection{Structure model and control model}

It will produce a man-machine interaction force when the movement of human body doesn't match completely with the HEE, and the goal of the control scheme is to reduce the energy consumption of human body by minimizing the interaction force $[13,14]$.

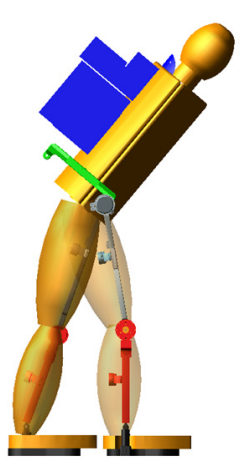

Fig. 1. Structure model

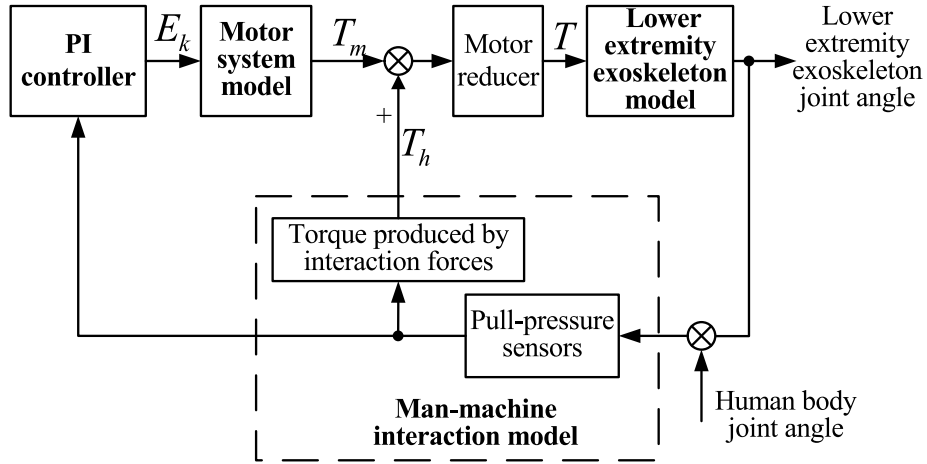

Fig. 2. Control model

\subsection{Simulation model and experimental scheme}

The MATLAB/Simulink simulation model is shown in Fig. 3. The experimental scheme is shown in Fig. 4, and it was conducted for the purpose of verifying the design of the whole electromechanical system of HEE and the simulation results [12].

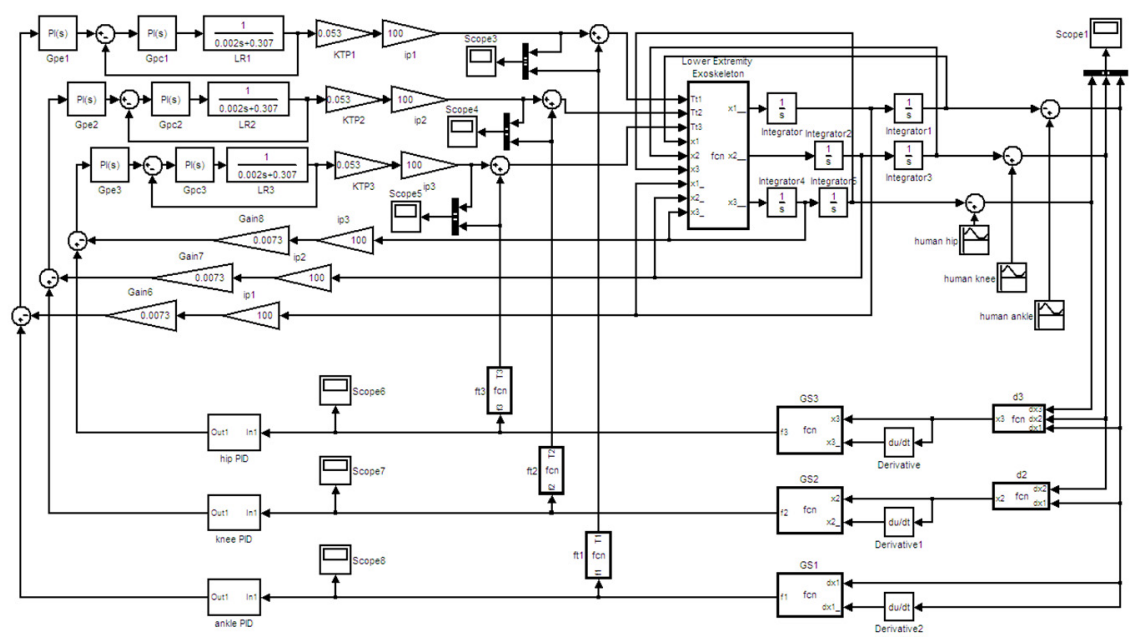

Fig. 3. MATLAB/Simulink simulation model

\section{Problem pretention}

Based on the following analysis (as in Section 3.1) of our previous simulation works and experiments, it can be concluded that the PI (Proportion Integration) controller parameters are not very appropriate for human body in every movement speed condition. So, the adaptability of the 
traditional PI controller is very necessary to be improved.

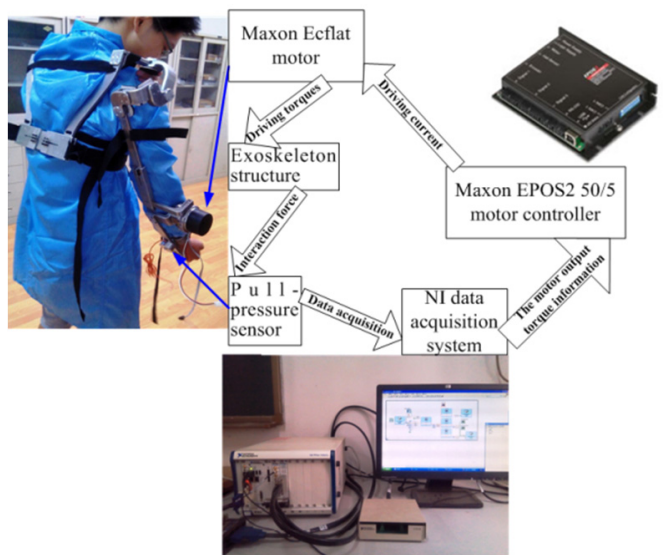

Fig. 4. The simplified experimental scheme

\subsection{Driving torque}

Obviously, most of the torques are provided by motors, so the power support effect of HEE can be proved effectively. However, because the parameters of the PI controller are set for the low speed condition, it can be seen by comparing Fig. 5 with Fig. 6 that the human body should provide much larger driving torques at high speed than that at low speed. So, it shows that the PI controller cannot obtain very good effect at high speed.

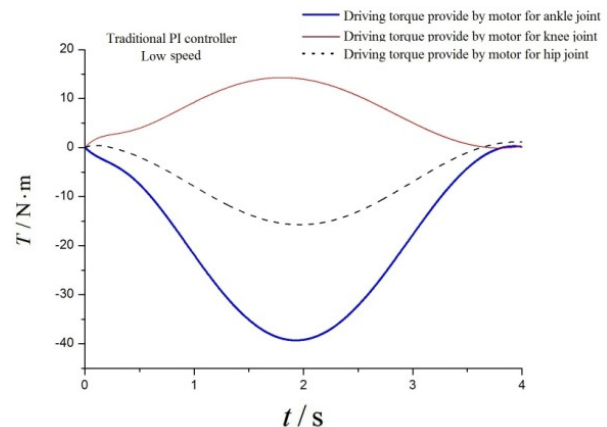

a)

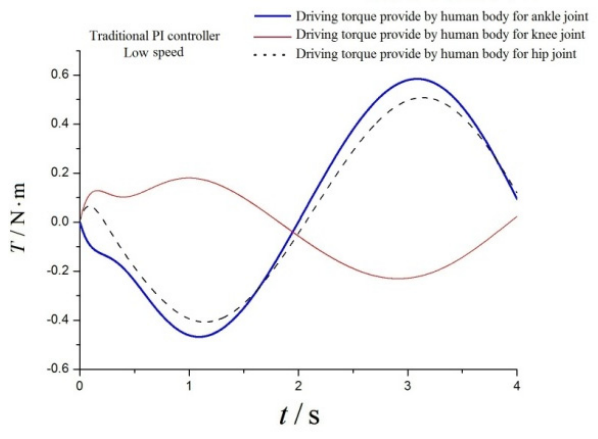

b)

Fig. 5. Driving torques at low speed a) by motor and b) human body

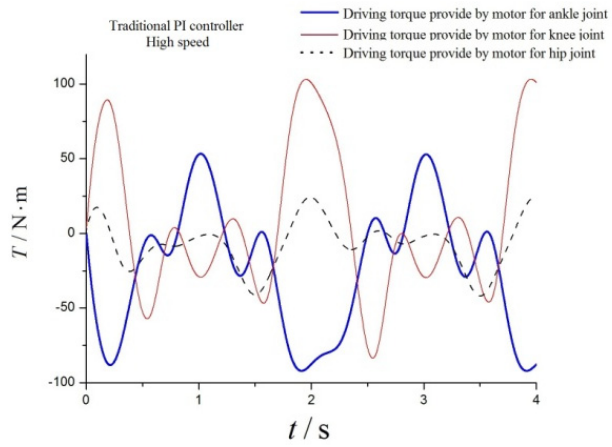

a)

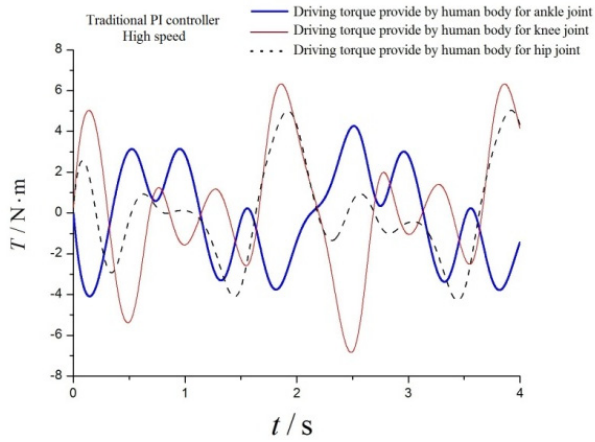

b)

Fig. 6. Driving torques at high speed a) by motor and b) human body 


\subsection{Angular deviation}

Because the parameters of the PI controller are set for the low speed condition, the angular deviations at low speed are always very small. In other words, the HEE can get good man-machine coordinated walking all the time at low speed. However, the angular deviations at high speed condition become much bigger, and it's unfavorable for the system security and performance.

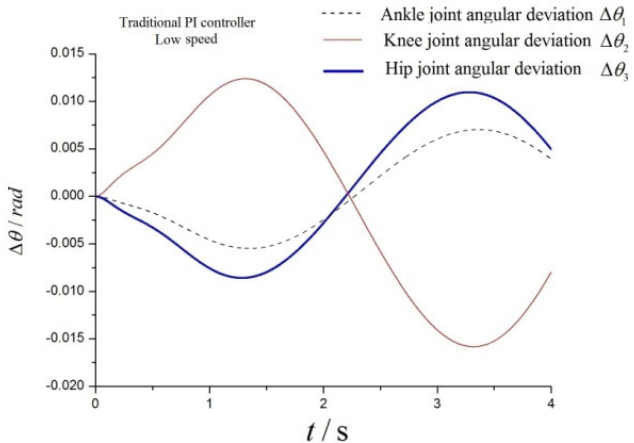

a)

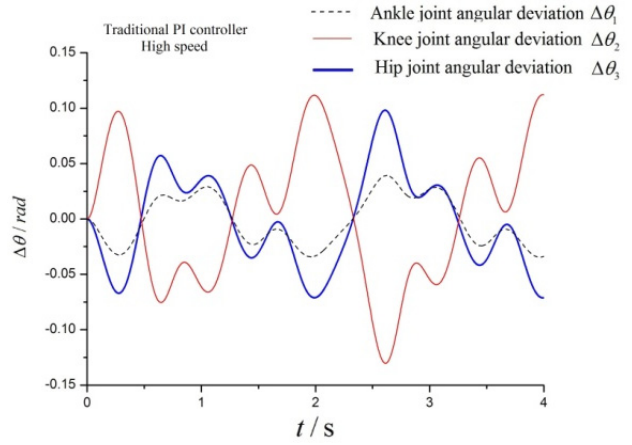

b)

Fig. 7. Angular deviations at a) low speed and b) high speed

\section{Optimization of the controller}

It can be seen from the above analysis that the traditional PI controller is not very appropriate for human body in every movement speed condition. So, one kind self-adaptive PI controller should be designed to make good man-machine coordinated walking can be got by the HEE at most conditions.

\subsection{Fuzzy self-adaptive PI controller model}

So, the fuzzy self-adaptive PI controller was proposed to solve this problem. The proportional-action coefficient $k_{p}$ and integral-action coefficient $k_{i}$ will be adjusted by the fuzzy controller based on the input signal e and input differential signal ec, so the PI controller can adapt different conditions [15]. The diagram of the fuzzy self-adaptive PI controller is shown in Fig. 8, the fuzzy self-adaptive PI controller subsystem simulation model in Simulink is shown in Fig. 9.

After processed by the Low-Pass Filter, the outputs of Fuzzy Logic Controller will be changed to the proportional-action coefficient $k_{p}$ and integral-action coefficient $k_{i}$. And the proportional integral (PI) signal can be obtained by multiplying the input signal and input Integral signal by $k_{p}$ and $k_{i}$.

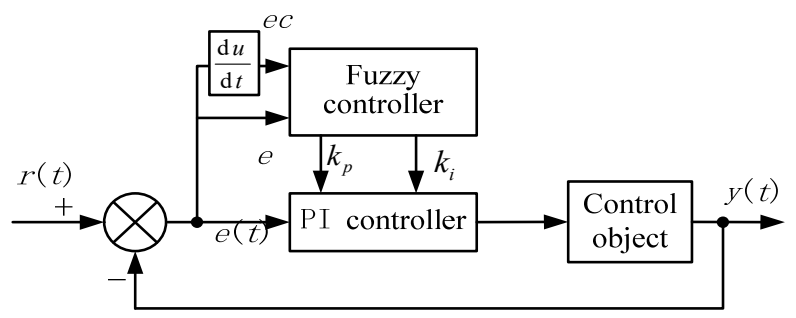

Fig. 8. Diagram of the fuzzy self-adaptive PI controller

\subsection{Parameters setting for fuzzy self-adaptive PI controller}

Input signal $e$; input differential signal $e c$; proportional-action coefficient $k_{p}$ and 
integral-action coefficient $k_{i}$ are all considered to obey normal distribution. The membership functions and fuzzy rules surfaces are shown in Figs. 10-12.

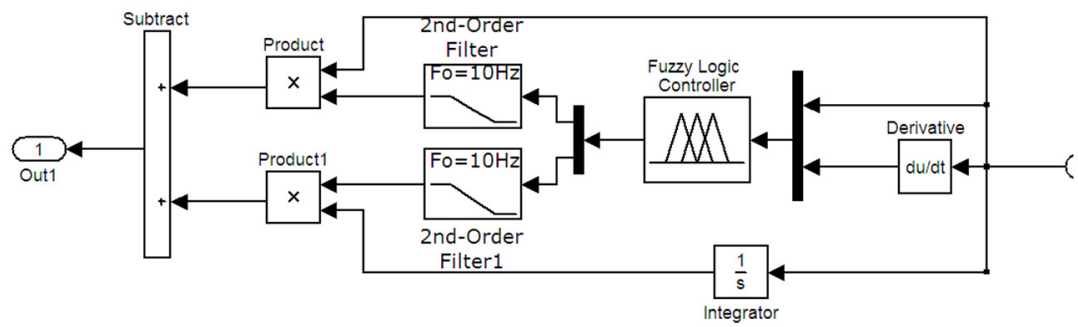

Fig. 9. Simulation model of fuzzy self-adaptive PI controller
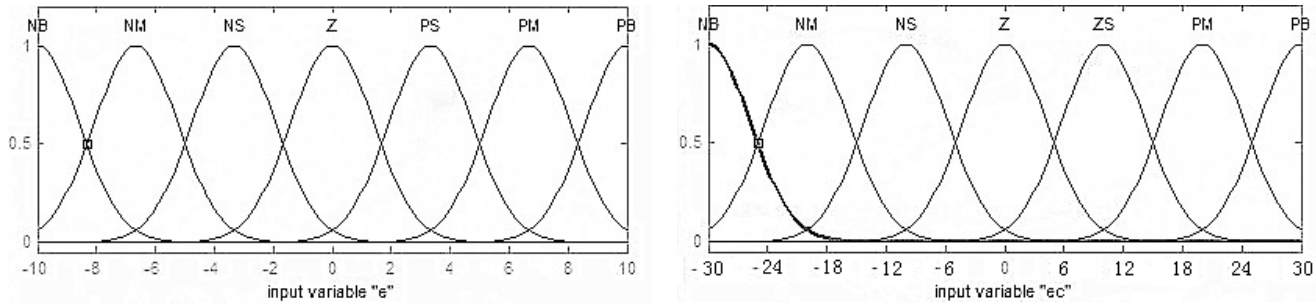

Fig. 10. Membership function of $e$ and $e c$
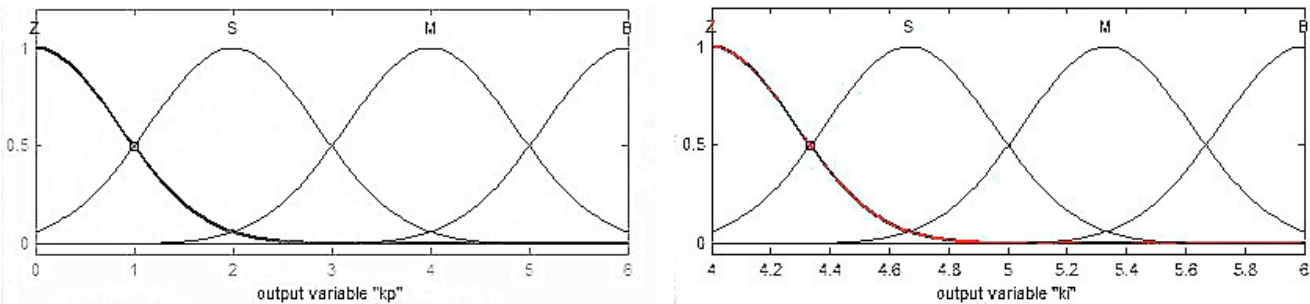

Fig. 11. Membership function of $k_{p}$ and $k_{i}$
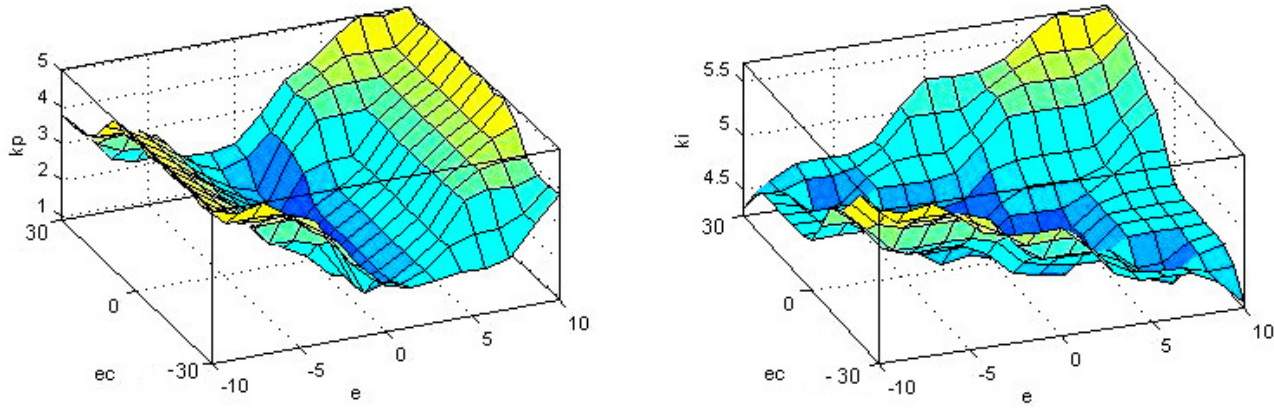

Fig. 12. Fuzzy rules surface of $k_{p}$ and $k_{i}$

\section{Simulation verification of the fuzzy self-adaptive PI controller}

\subsection{Driving torque}

Only the traditional PI controller of Fig. 2 was changed to fuzzy self-adaptive PI controller, and the driving torques which are calculated out by simulation study are shown in Figs. 13-14.

Based on the figures, it can be concluded that the motors' driving torques didn't change much after the fuzzy controller was added to the traditional PI controller. However, the driving torques by human body at high speed decreased greatly after the fuzzy controller was added to the 
traditional PI controller. So, it proves the fuzzy self-adaptive PI control scheme can achieve very good assisted effect at any condition. Besides, the total torque at high speed decreased slightly, so it means the fuzzy self-adaptive PI controller has higher energy efficiency.
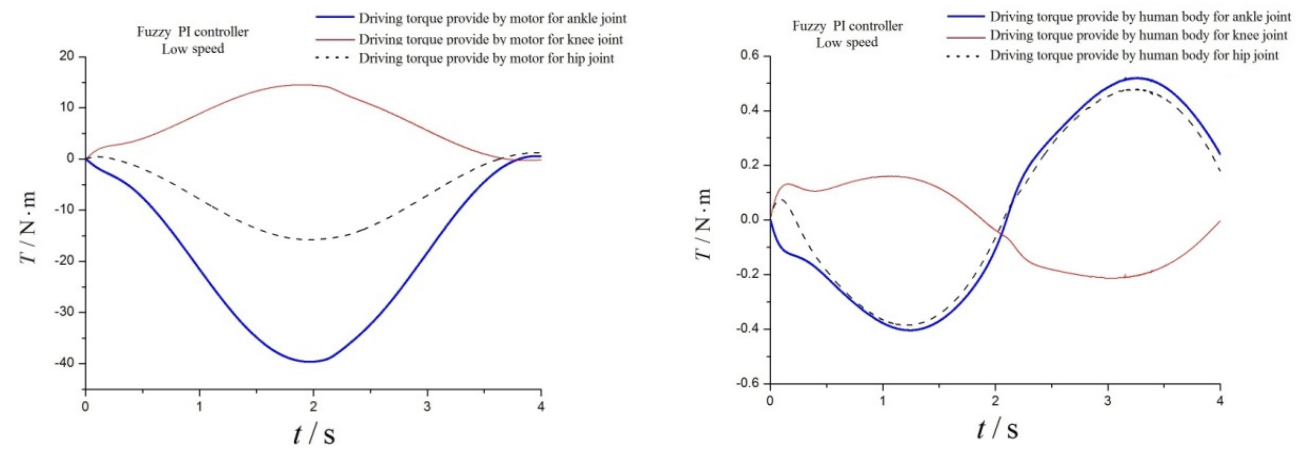

Fig. 13. Driving torques at low speed by motor and human body
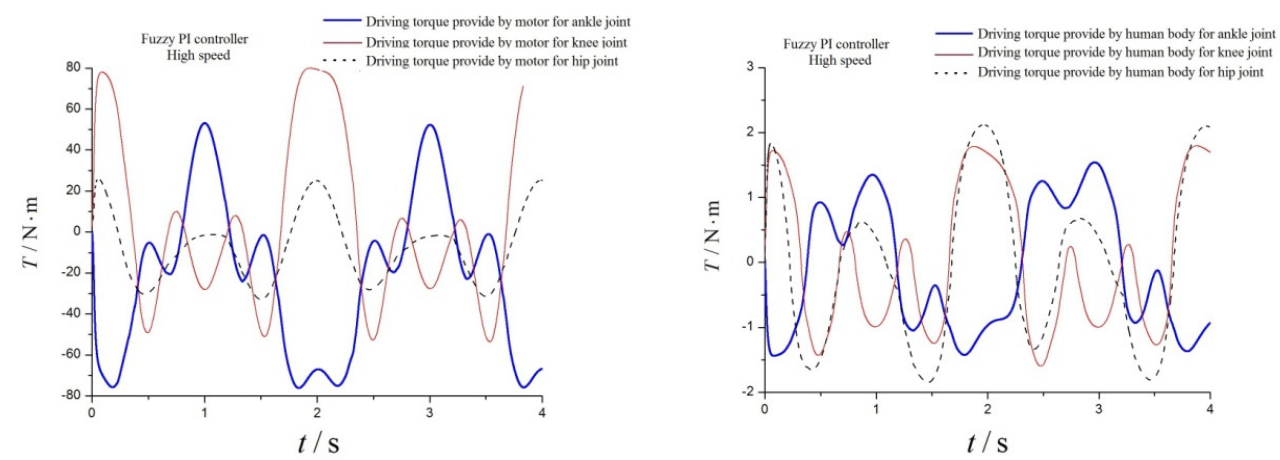

Fig. 14. Driving torques at high speed by motor and human body

\subsection{Angular deviation}

Only the traditional PI controller of Fig. 2 was changed to fuzzy self-adaptive PI controller, the obtained angular deviations are shown in Fig. 15.

Based on Fig. 15, it can be concluded that all the angular deviations keep very small no matter at high speed condition or low speed condition. So, it can be analyzed out that the human body can be tracked very well by the HEE at both high and low speed condition. Therefore, it proves that good man-machine coordinated walking can be achieved by the control scheme at any condition.
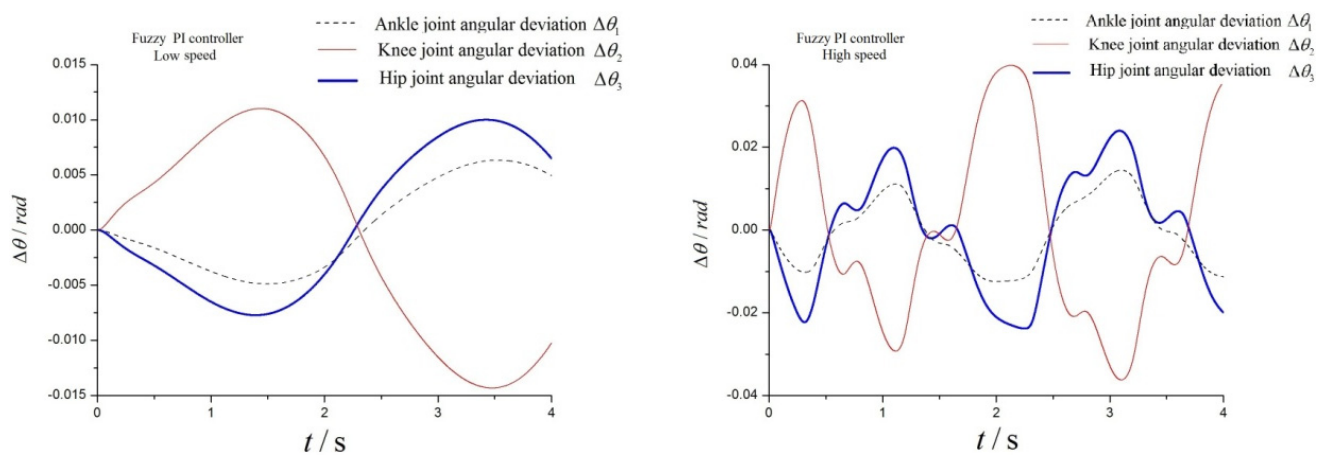

Fig. 15. Angular deviations at low speed and high speed 


\section{Experimental verification of the fuzzy self-adaptive PI controller}

The experimental scheme (as shown in Fig. 4) is still adopted here.

Based on Fig. 16, it is obvious that the angular deviation of elbow joint didn't change much after the fuzzy controller was added to the traditional PI controller at low speed but greatly reduced at high speed. So, just the same as the simulation results, it proves that good man-machine coordinated walking can be achieved by the fuzzy self-adaptive control scheme no matter at high speed condition or low speed condition.

And based on wearer's experience, the movement process can be easily carried out when the fuzzy controller was added to the traditional PI controller at high speed. In contrast, if the fuzzy controller was removed, it will become much harder for the wearer to carry out the movement process.
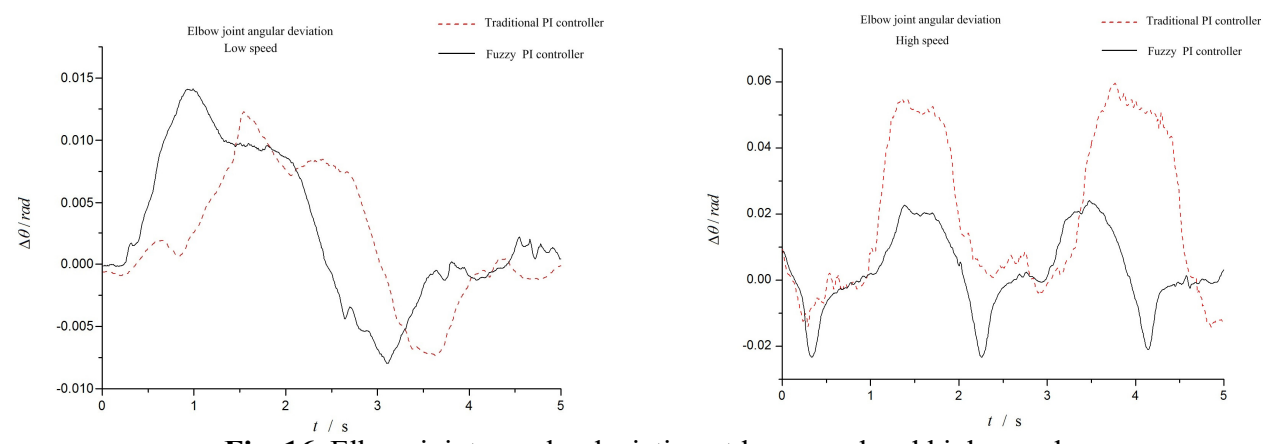

Fig. 16. Elbow joint angular deviation at low speed and high speed

\section{Conclusion}

For the purpose of designing a suitable control scheme for HEE, in our previous work, one kind traditional PI control scheme was proposed. And by analyzing the previous simulation and experiment results, the main problem can be found that the traditional PI controller is not very appropriate for human body in every movement speed condition.

So, in this paper, the fuzzy self-adaptive controller was added to the traditional PI controller to solve this problem. And then, the improved simulation and experiment were conducted. The simulation and experimental results show that the motors' driving torques are much bigger than those driving torques provided by human body. Besides, angular deviations at both low speed condition and high speed condition are all acceptable. So, it proves that the fuzzy self-adaptive control scheme can achieve the purpose of our optimization.

\section{Acknowledgements}

The work reported in this paper is funded by the National Natural Science Foundation of China (No. 51575279).

\section{References}

[1] Yang Zhiyong, Gu Wenjing, Zhang Jing, Gui Lihua Force Control Theory and Method of Soldier Load Carrying Exoskeleton Suit. National Defense Industry Press, 2013, p. 1-5.

[2] Anam K., Al-Jumaily A. A. Active exoskeleton control systems: state of the art. Procedia Engineering, 2012, p. 988-994.

[3] Zoss A., Kazerooni H. On the mechanical design of the Berkeley lower extremity exoskeleton (BLEEX). Proceedings of IEEE Intelligent Robots and Systems, Edmonton, Canada, 2005, p. 3132-3139.

[4] Zoss A., Kazerooni H., Andrew Chu On the biomechanical design of the Berkeley lower extremity exoskeleton (BLEEX). IEEE/ASME Transactions on Mechatronics, Vol. 11, Issue 2, 2006, p. 128-138. 
[5] Kazerooni H., Racine Jean-Louis, Huang Lihua, Steger Ryan On the control of Berkeley lower extremity exoskeleton (BLEEX), Proceedings of IEEE International Conference on Robotics and Automation, Barcelona, Spain, 2005, p. 4364-4371.

[6] Tomoyoshi Kawabata, Hozumi Satoh Working posture control of robot suit HAL for reducing structural stress. IEEE International Conference on Robotics and Biomimetics, Guilin, China, 2009.

[7] Esquenazi A., Talaty M., Packel A., et al. The ReWalk powered exoskeleton to restore ambulatory function to individuals with thoracic-level motor-complete spinal cord injury. American Journal of Physical Medicine and Rehabilitation, Vol. 91, Issue 11, 2012, p. 911-921.

[8] Low K. H., Liu Xiaopeng, Yu Haoyong Development of NTU wearable exoskeleton system for assistive technologies. IEEE International Conference on Mechatronics and Automation Niagara Falls, Canada, 2005, p. 1099-1106.

[9] Long Y., Du Z. J., Wang W. D., et al. Robust sliding mode control based on GA optimization and CMAC compensation for lower limb exoskeleton. Applied Bionics and Biomechanics, Vol. 2016, 2016.

[10] Li Yang, Guan Xiaorong, Xu Cheng Dynamics simulation of lower extremity exoskeleton based on human gait. Journal of Nanjing University of Science and Technology, 2015, p. 353-357.

[11] Li Zhengyi, Cao Huimin Robot impedance control method adapting to unknown or changing environment stiffness and damping parameters. China Mechanical Engineering, 2014, p. 1581-1585.

[12] Li Y., Guan X., Tong Y., et al. Design and simulation study of the translational-knee lower extremity exoskeleton. Mechanika, Vol. 21, Issue 3, 2015, p. 207-213.

[13] Li Y., Guan X., Xu C. Research of force amplification control strategy of lower extremity exoskeleton. 5th International Workshop on Computer Science and Engineering: Information Processing and Control Engineering, Moscow, Russia, 2015.

[14] Li Yang, Xu Cheng, Guan Xiaorong Modeling and simulation study of electromechanically system of the human extremity exoskeleton. Journal of Vibroengineering, 2016, p. 551-561.

[15] Ying Wu, Hang Jiang, Min Zou The research on fuzzy PID control of the permanent magnet linear synchronous motor. Physics Procedia, Vol. 24, 2012, p. 1311-1318.

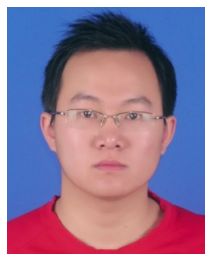

Yang Li received B.E. degree in Nanjing University of Science and Technology, Nanjing, China, in 2012. Now he is studying for engineering Ph.D. in Nanjing University of Science and Technology. His current research interests include dynamics, control and human extremity exoskeleton.

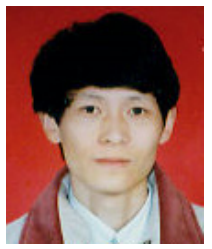

Cheng Xu received Ph.D. degree in Nanjing University of Aeronautics and Astronautics, Nanjing, China. He has worked at Stanford University and TU Berlin for several years. Now he is a Professor of Nanjing University of Science and Technology. His current research interests include dynamics, small arms and human extremity exoskeleton.

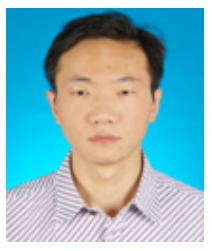

Xiaorong Guan received Ph.D. Degree at Nanjing University of Science and Technology, China. He has worked at Tsinghua University for several years. Now, he is a teacher of Nanjing University of Science and Technology. His Current research interests include structural dynamics, mechanical and electrical system, and human extremity exoskeleton.

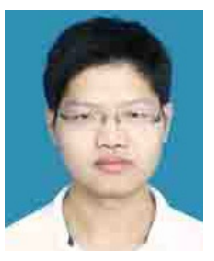

Zhong Li received B.E. degree in Nanjing University of Science and Technology, Nanjing, China, in 2014. Now he is studying for engineering Ph.D. in Nanjing University of Science and Technology. His current research interests include dynamics simulation and human extremity exoskeleton. 